\title{
Effects of form rotation on the speed of classification: The development of shape constancy
}

\author{
DAVID SHINAR and DEAN H. OWEN* \\ The Ohio State University, Columbus, Ohio 43210
}

\begin{abstract}
Ss classified a test form as matching or not matching one of a set of memorized forms relative to which the test forms were rotated up to $90 \mathrm{deg}$. "Match" reaction times increased monotonically with both number of forms memorized and degrees of rotation. Disappearance of the rotation effect after practice was attributed to a change from considering rotational information during comparison to comparing rotation invariant features. The change in strategy is considered an indicant of the development of shape constancy. Time taken to memorize the patterns increased linearly with the size of the memory set, justifying interpretation of the RT effects in terms of comparison time differences rather than differences in memorial specification of the patterns.
\end{abstract}

People are noted for their ability to recognize objects under a wide variety of transformations. One commonly occurring transformation is a change in orientation, the rotation of a pattern or an object around an arbitrary point, for example, its center of gravity. A long history of experimental studies has evidenced the detrimental effects of rotation on pattern recognition (see Hake, 1957, for a review). Woodring and Alluisi (1966), for example, found that both latency of response and error rate in a recognition task increased with rotated patterns. However, the experimental task they used did not enable them to study separately the differential effects of the degrees of rotation. These effects were measured in an experiment by Shepard and Metzler (1971). In this study, perspective drawings of three-dimensional objects were presented in pairs, and the $S$ had to judge whether the two pictures were of the same object or not. They found that the time to make a correct "same" judgment increased linearly with the angle of rotation at the rate of approximately $17 \mathrm{msec} / \mathrm{deg}$, and suggested that the $\mathrm{Ss}$ in this task performed an operation which they characterized as "mental rotation" of one of the two forms until a match was achieved.

While these studies demonstrated the effects of rotation on recognition and discrimination, they did not lead to any explanation of the underlying processes responsible for these effects, especially in the case of recognition, that is, what is the nature of the interaction between the perceived information that specifies a shape and the memorial information that specifies that shape? It is of interest, then, to consider the nature of the processes involved in pattern recognition when the orientation of the test pattern differs from the orientation of the pattern memorized. Possible processing stages involved in a pattern classification task have been extensively studied by Sternberg (cf. 1969).

\footnotetext{
*Requests for reprints should be addressed to Dean $\mathrm{H}$. Owen, Department of Psychology, The Ohio State University, 404-C West 17 th Avenue, Columbus, Ohio 43210.
}

According to his model, a test pattern is first preprocessed, then the preprocessed pattern is compared serially to each one of the possible memorized patterns. Following an exhaustive series of such comparisons, a decision is made as to whether the test pattern matches one of the memorized patterns or not. Given these processing stages, recognition of a rotated pattern may be achieved in one of two ways: One possibility is that the orientation of the test pattern is somehow dealt with during the preprocessing stage, that is, the test pattern is "cleaned up" (Sternberg, 1967) and only then compared to the memorized patterns to test for recognition. Such a strategy requires that the perceiver be familiar enough with the population of patterns to know how to normalize the test pattern information prior to its comparison with any memorial information. Another possibility is that the perceiver takes rotational differences into account while comparing the test pattern with each of the memorized patterns until a match occurs.

To determine the locus of rotation effects, Sternberg's (1967) classification task was employed. The procedure required a $S$ to judge whether a test pattern matched one of a number of memorized patterns. Given that the test pattern was rotated in relation to the memorized pattern and that rotation was irrelevant to the task, RT was expected to increase with increasing degrees of rotation. The value of the Stemberg paradigm lies in the possibility of localizing the increase in $R T$ as an influence on a precomparison activity vs the comparison activity itself. According to Sternberg's assumptions, if the additional time to recognize a pattern reflects a precomparison activity, then the time increment should be independent of the number of memorized patterns with which the test pattern is compared. A precomparison influence would result in an increase in the intercept of the function relating RT to number of patterns memorized, as Sternberg (1967) observed after superimposing a noise field on the test pattern. If, on the other hand, rotation of the test pattern increases the duration of its comparison with each memorized 

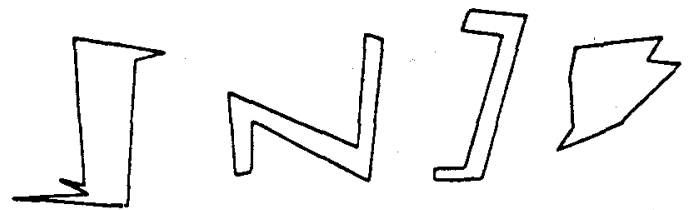

load, RT still increases with set size, then the increase can be attributed to influence of a stage as opposed to differences in "strength" of representations.

\section{METHOD}

\section{Procedure and Stimuli}

The $S$ sat in a dimly lighted booth facing a round translucent screen, which subtended $3 \mathrm{deg}$ and $35 \mathrm{~min}$ of visual angle. Problems were constructed from eight different Attneave and Arnoult (1956) Method I eight-sided patterns with equal area (see Fig. 1), each subtending approximately $35 \mathrm{~min}$ of visual angle. The authors selected the patterns from a larger sample on the basis of high judged dissimilarity among the patterns in all orientations used. On each trial, a set of $1,2,3$, or 4 patterns was presented simultaneously, centered on the screen in a horizontal array. As soon as the $S$ felt that she had the set committed to memory, she extinguished the display by pressing a footpedal. There followed a 2 -sec interval, which ended with the onset of a single test pattern. The S's task was to move a lever on the armrest of her chair to the right or left, depending on whether the test pattern belonged to the memorized set or not. The Ss were instructed to respond as quickly as possible without making errors. The intertrial interval was approximately $10 \mathrm{sec}$. Memorization time (MT) from onset of the patterns to be memorized to its offset by $\mathrm{S}$ and $\mathrm{RT}$ from onset of test pattern to initiation of the choice response were recorded on each trial. Frror RTs and frequencies were recorded, and error trials were recirculated in the same session.

\section{Design}

There were three independent variables: (a) memory load (M) - number of patterns in the memorized set; (b) orientation - the test pattern on each trial could appear in the same orientation as during the positive set presentation $(0 \mathrm{deg})$ or in one of three clockwise orientations: 30,60 , or $90 \mathrm{deg}$; (c) set membership-the test pattern either matched one of the memorized patterns or did not match any of them on a given trial.

Each block of trials consisted of one complete permutation of test pattern (8) by set membership (2) by orientation of the test pattern (4), totaling 64 trials. Each S participated in 10 blocks, 1 practice and 9 experimental. In the practice block, memory load was one pattern for all Ss. The purpose of this block was to familiarize the $S$ with the patterns, the task, and the extent of the rotation transformation. In each block, $S$ was presented with each pattern eight times-once as a test pattern that had been memorized and once as a test pattern that had not been memorized-in each orientation.

In the experimental blocks, memory load was 2,3 , or 4 patterns, and was constant for a given block, but the order of the memory loads was counterbalanced for every three blocks across Ss and across blocks within a S. The nine blocks were distributed over 4 consecutive days of testing, with the first 10 trials each day serving as a warmup.

\section{Apparatus}

Two random-access projectors were used to display the memorization set slide and the test pattern slide on each trial. An interval timer controlled the 2-sec delay between the offset of the memorized set and the onset of the test pattern. A frequency counter accumulated separately the S's memorization time and the classification in $\mathrm{RT}$ in milliseconds. Ss received white masking noise at $85 \mathrm{~dB}$ through headphones to reduce interference from extraneous sounds. 
Subjects

Twenty-four female Ohio State University undergraduates served as Ss in fulfillment of a course requirement.

\section{RESULTS}

Memorization time, correct RT, and errors were analyzed separately for the practice block $(M=1)$ and for the nine experimental blocks $(M=2,3$, or 4$)$. For statistical analysis, mean times, median times, and total number of errors were computed for each combination of Orientation by Set Membership in each block. Thus, each mean and median is based on eight trials with all the different test patterns. In most cases, the mean and median MTs and RTs yielded similar results and therefore, except for those cases where the results with the two measures diverge, discussion will be limited to the effects on the means, considered by Sternberg (1969) to be the more appropriate dependent measure.

\section{Practice Block, $M=1$}

Memorization times in this block were relatively long (MT $=991 \mathrm{msec}$ ), reflecting the initial stage of learning when the Ss were still unfamiliar with the set of patterns. Recognition RTs were subjected to a Set Membership by Test Pattern Orientation analysis of variance. Results showed that nonmatch responses were slightly, but significantly, faster than match responses, $F(1,30)=7.70, p<.025$. The main effect of orientation was not significant, $F(3,69)=2.39, p>.05$, but the interaction between the two variables was, $F(3,69)=$ $4.33, p<.01$. This interaction is displayed in Fig. 2, from which it can be seen that test pattern orientation affected the match responses only, evidenced by a monotonic increase in RT as the angular disparity between the orientation of the pattern memorized and that of the test pattern increased $(1.38 \mathrm{msec} / \mathrm{deg})$. This effect was slightly more systematic for the median RTs.

Overall error rate was $2.2 \%$. Error rate was slightly, but not significantly, $F(1,23)=2.53, \mathrm{p}>.10$, higher for the match trials and showed a slight, but nonsignificant, $\mathrm{F}(3,69)<1.00$, tendency to increase with orientation on the match trials $(2.1 \%, 2.1 \%, 3.1 \%$, and $3.9 \%$ for 0 , 30,60 , and $90 \mathrm{deg}$, respectively).

\section{Experimental Blocks, $M=2,3,4$}

Both MTs and RTs were subjected to separate memory load (3) by set membership (2) by practice (3) by orientation (4) analyses of of variance. The analysis of MTs revealed that the most significant effect was that of memory load, $\mathrm{F}(2,46)=187.30, \mathrm{p}<.001$; the average time to commit two patterns to memory was $1,225 \mathrm{msec}$, and that time increased linearly with the number of patterns memorized at the average rate of $800 \mathrm{msec}$ per pattern. The effect of practice was to decrease MT, as evidenced by the significant Memory

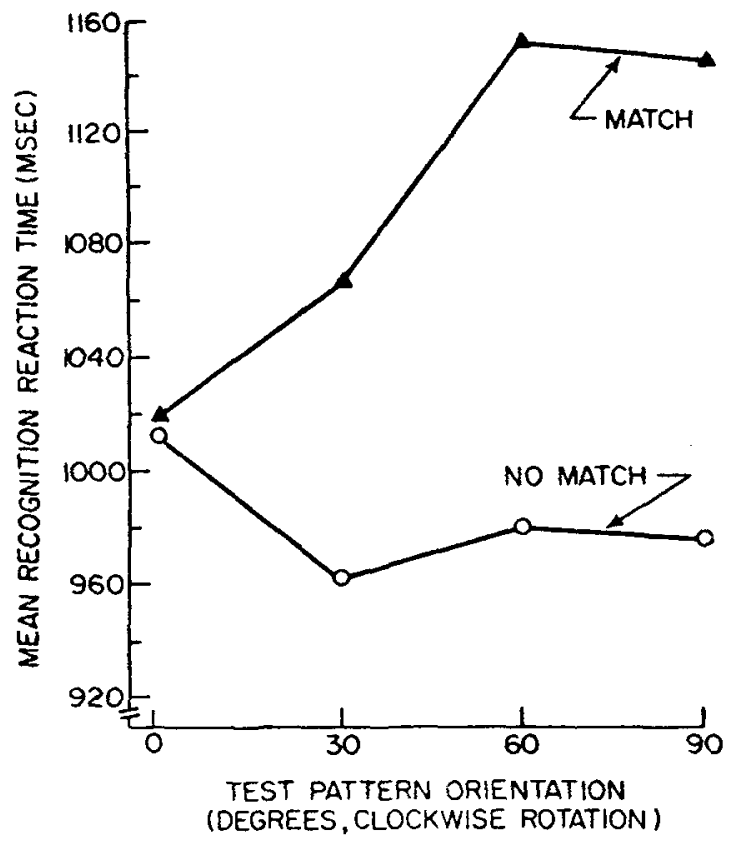

Fig. 2. Recognition reaction time as a function of test pattern orientation and set membership for the practice session with one pattern memorized.

Load by Practice interaction (in sessions of three consecutive blocks, each with a different memory load), $F(4,92)=3.00, p<.05$.

Excluding orientation, all main effects of the RT analysis were significant at the .001 level. Mean RT increased with the memory load at the rate of $100 \mathrm{msec} /$ pattern, $\mathrm{F}(2,46)=36.17$, was $65 \mathrm{msec}$ faster for the match responses, $F(1,23)=31.07$, and decreased with practice, $F(2,46)=56.26$. As noted, the main effect of orientation was not significant, $F(3,69)=2.01$, $p>.05$. Likewise, the interaction of Orientation by Memory Load was not significant, $F(6,138)=1.80$, $p>.05$. However, orientation interacted significantly with each of the other independent variables alone and with all the variables together. The last was a significant, $\mathrm{F}(12,276)=2.18, \mathrm{p}<.05$, four-way Memory Load by Orientation by Set Membership by Practice interaction. Due to this interaction with practice, a separate analysis of variance was performed for each of the three sessions. These analyses revealed that the effect of orientation was limited to the first session, where the main effect, $F(3,69)=3.46$, and more importantly, the interaction of orientation with set membership, $F(3,69)=6.64$, were significant at the .05 and .01 levels, respectively. Neither of these effects was significant in Sessions 2 and 3 . These relationships are graphically presented in Fig. 3 . The effects of rotating the test pattern are well defined: RT increased (at the rate of $1.11 \mathrm{msec} / \mathrm{deg}$ ) only when the test pattern matched one of the positive set items and then only when the patterns were still relatively unfamiliar. In addition to an overall decrease in RT, the effect of practice was to nullify the increased RT due to 


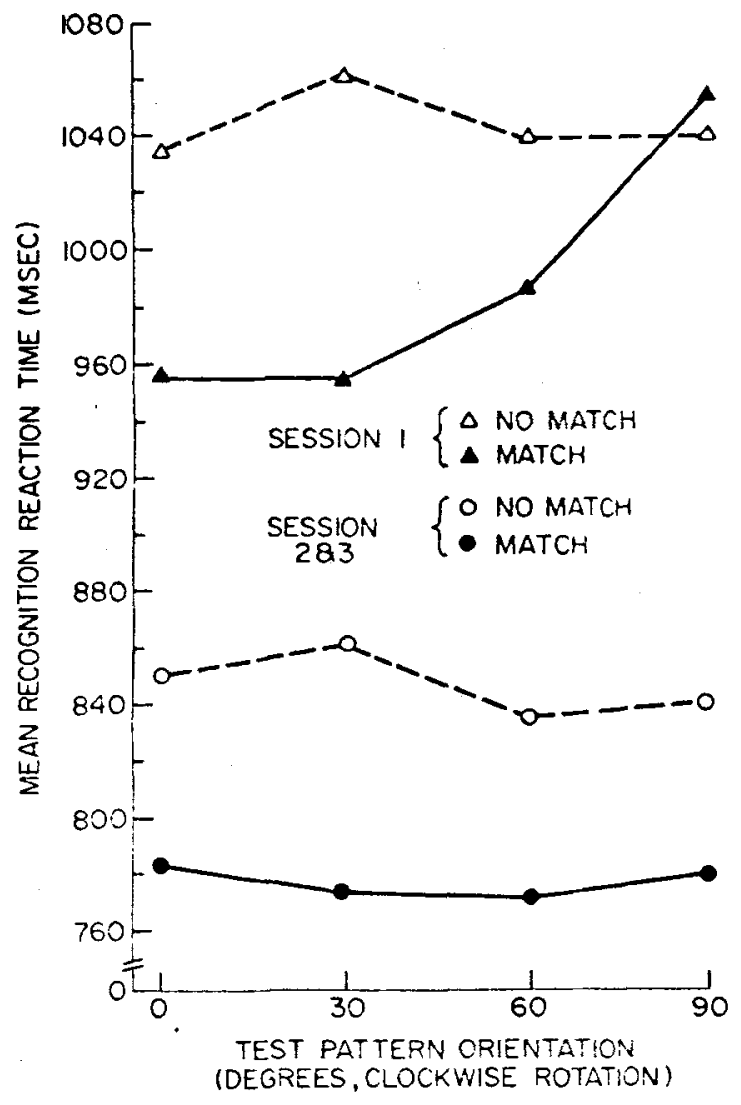

Fig. 3. Mean recognition reaction time for each level of test pattern orientation as a function of practice and set membership.

rotating the test pattern more than $30 \mathrm{deg}$. In Session 1 , the differential effect of orientation on the set membership was statistically supported by the three-way Orientation by Memory Load by Set Membership interaction. $F(6,138)=2.85, \mathrm{p}<.05$.

The only variable other than orientation that interacted significantly with practice was memory load, $F(4,92)=3.00, p<.05$. The decrease in RT with practice as a function of memory load and orientation is graphed in Fig. 4. In this figure, only match responses are represented, since it was shown above (Fig. 3) that only match times were sensitive to the rotation transformations. Because the effect of orientation was significant only for the first session, only the means for this session are graphed separately for each orientation. Two points are worth noting in Fig. 4: First, in Session 1, the effect of rotation seems to be almost exclusively a slope effect; increasing the rotation increased the comparison time by $13 \mathrm{msec}$ per memorized pattern when the rotation was increased from 30 to $60 \mathrm{deg}$ and by an additional $27 \mathrm{msec}$ when it was further increased to $90 \mathrm{deg}$. The relationship was very systematic for all levels of orientation above $0 \mathrm{deg}$. At $0 \mathrm{deg}$, the RT points deviate markedly from a linear fit as a function of the memory load. Secondly, the effect of practice in this task was to reduce the slope constant by a third from the first to the last session without affecting the intercept at all.

Overall error rate was $3.0 \%$. Error rate increased slightly, though significantly, as a function of memory load, $\mathrm{F}(2,46)=13.90, \mathrm{p}<.001,1.6 \%, 3.0 \%$, and $4.6 \%$ for $M=2,3$, and 4 , respectively. The decrease in error rate with practice was also significant, $F(2,46)=10.70$, $p<.001$. Orientation, as a main effect or at any level of interaction, did not affect the error rate significantly. Most critical in terms of speed/accuracy considerations are the error rates for match responses in Session 1, during which orientation had a significant effect on RT. These are given in Table 1.

\section{DISCUSSION}

\section{Memorization Time}

Unlike previous experiments in which the Sternberg classification task was used with exposure duration of the memory set as a fixed parameter, in this study, memory set exposure duration was a dependent variable. The results showed that time taken to memorize a set of patterns increased linearly with set size and decreased with practice. If MT is assumed to be a measure of information pickup time, then the procedure of memory experiments should be self-paced, or at least allow equal MT per item, so that memorial specification of patterns

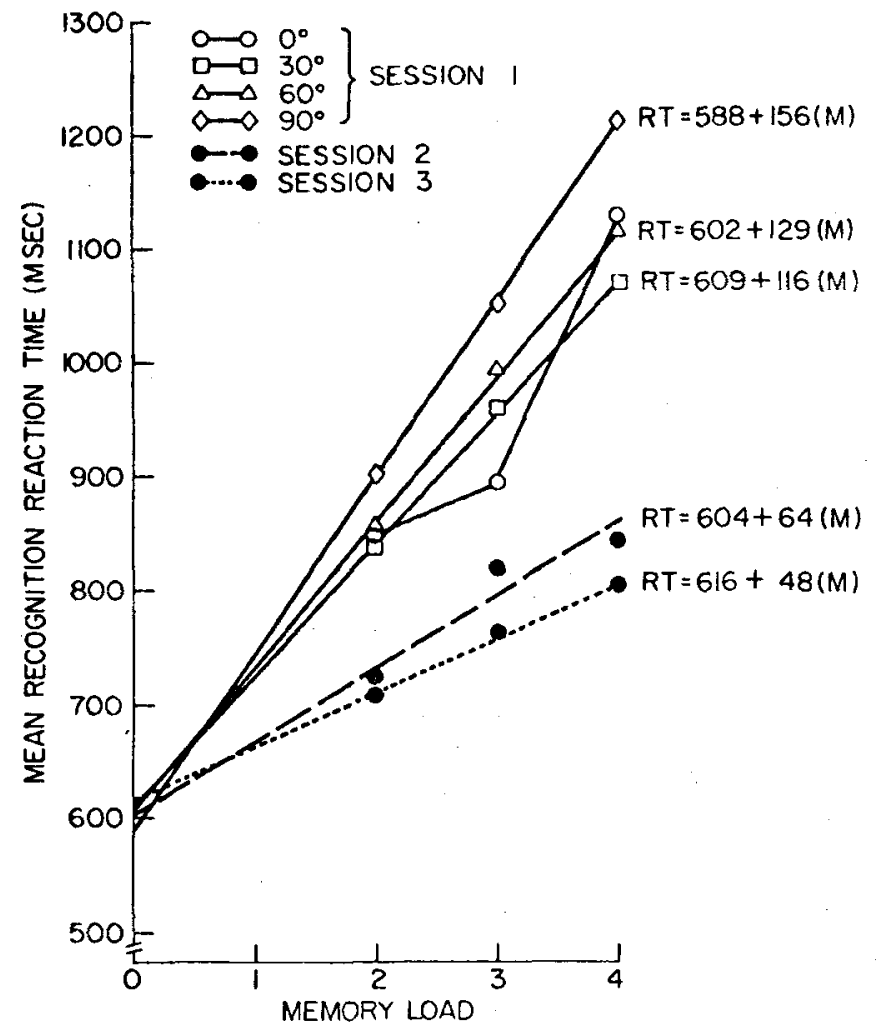

Fig. 4. The effect of test pattern orientation on mean reaction time for the match trials as a function of memory load and practice at the task. 
is the same across all levels of memory load. The linear increase in MT with memory set size favors the interpretation of an RT effect with increased memory load in terms of increased comparison time rather than differences in "trace strength" or extent of pattern specification.

\section{Reaction Time}

The results of this study demonstrated a transient effect of rotation on latency of pattern recognition. With a limited population of highly discriminable forms, rotational effects were monotonically related to the difference in orientation only at the initial stages of practice at the task (practice and Session 1). ${ }^{1}$ Although transient, the effect is real, and not due to a change in the speed/accuracy tradeoff across different levels of orientation. This is evident from the increase in the error rate as a function of orientation in the practice session and the relative stability of errors across levels of orientation in Session 1.

The manifestation of the orientation effects in the slope of the function relating RT to memory load, coupled with the Orientation by Memory Load and Orientation by Set Membership interactions, suggests that orientation affected the speed of comparison. The rotation transformation has, therefore, a different influence than the degradation caused by a noise field, an effect which has been localized in a precomparison stage (Sternberg, 1967).

Considered in terms of the function relating RT to memory load, the common intercept for the different levels of orientation (Fig. 4, Session 1) suggests that precomparison activities were the same for test patterns in all orientations. The difference in slopes, however, suggests that rotation-dependent information was considered in the comparison stage. Further, the increase in slope with increase in orientation (over $30 \mathrm{deg}$ ) is consistent with the hypothesis that rotation-dependent differences might be tested during comparison. Finally, the results in Fig. 3 indicate that the process of attending to rotation-invariant features appears to be a self-terminating one in the sense that a match response is initiated as soon as a match is found, while a no-match response is made only after all testing for a match has failed.

The picture changed for the results of the second and third sessions, for which there was evidence that the speed of comparison increased with practice while the effect of rotation disappeared completely. Thus, there was a different effect of practice for two variables presumed to influence the comparison. While the increase in the speed of comparison with practice has been noted before (see, for example, Checkosky, 1971), the total disappearance of the effect of orientation was, in one sense, unusual. If, with increased familiarity, rotational differences were handled in a precomparison stage, then the intercept value of the RT function should
Table 1

Percent Errors in Session 1 as a Function of Memory Load, Orientation, and Set Membership

\begin{tabular}{|c|c|c|c|c|c|c|c|c|}
\hline \multirow{2}{*}{$\begin{array}{c}\text { Memory } \\
\text { Load }\end{array}$} & \multicolumn{4}{|c|}{ Match Trials (Deg) } & \multicolumn{4}{|c|}{ No-Match Trials (Deg) } \\
\hline & 0 & 30 & 60 & 90 & 0 & 30 & 60 & 90 \\
\hline $\begin{array}{l}2 \\
3 \\
4\end{array}$ & $\begin{array}{l}2.1 \\
1.0 \\
4.7\end{array}$ & $\begin{array}{l}3.1 \\
3.6 \\
7.3\end{array}$ & $\begin{array}{l}1.6 \\
5.1 \\
5.7\end{array}$ & $\begin{array}{l}2.1 \\
3.1 \\
6.3\end{array}$ & $\begin{array}{r}2.1 \\
5.2 \\
10.1\end{array}$ & $\begin{array}{l}3.1 \\
5.7 \\
8.3\end{array}$ & $\begin{array}{l}1.6 \\
6.8 \\
7.8\end{array}$ & $\begin{array}{l}2.1 \\
3.6 \\
8.3\end{array}$ \\
\hline
\end{tabular}

have increased. The total disappearance of the rotation effect, coupled with the decrease in the slope of the RT function, suggests that the effect of practice was a qualitative one: The practiced Ss extracted from each pattern some information that was invariant across all orientations, that is, in the second and third sessions, the effective information was no longer rotation specific but rather some rotation-invariant features with which comparisons were then made.

While the results of Session 1 support Sternberg's (1967) argument for matching based on physical properties of the stimulus, the disappearance of all orientation effects suggests that there might have been a change to a recoding strategy. This explanation is consistent with recent theoretical formulation (Atkinson \& Shiffrin, 1968) and experimental results (Posner, Boies, Eichelman, \& Taylor, 1969; Tversky, 1969) suggesting that recoding may be under the S's voluntary control. In particular, Wattenbarger (1970) has made a strong argument for verbal recoding and comparison in a Sternberg task. In the debriefing that followed the last session of the present experiment, all Ss without exception noted that they assigned each of the forms a verbal label, and most Ss reported that this labeling was completed by the end of the first session. It is interesting to note that in a completely different experimental task, Tversky (1969) found that identification latencies of highly familiar names did not increase when the names were presented in a tilted form. Lack of an effect of rotation on either identification time or classification time would indicate that if verbal codes were used, either they were associated with rotation-invariant perceptual information or the common verbal label associated with each orientation of the pattern served as the invariant.

The experiment was designed to allow definitive outcomes relative to the problem of localizing rotation effects and was successful in that respect. The finding that rotation differences were considered during comparison does not, however, lead to a definitive explanation of how the comparison activity was influenced. Two different theoretical alternatives will be considered; first, the "mental rotation" process proposed by Shepard and Metzler (1971), and second, the notion that the rotational transformation provides information indicating that the memorized and test configurations of a rotated pattern are not the same.

As noted earlier, a linear increase in "same" judgment time with increasing difference in orientation suggested 
to Shepard and Metzler that Ss were mentally rotating one of the forms until the two configurations matched. The present results differ from those obtained by Shepard and Metzler in several ways. In their study, the size of the orientation effect was larger by an order of magnitude. the function relating RT to degrees of rotation was highly linear, and no decrease in the effect with increasing practice was noted. Thus, the influence on comparison time of a change in orientation in this study probably reflects a different process from that of Shepard and Metzler's mental rotation.

With reference to judgments of sameness, Woodring and Alluisi (1966) considered rotational differences as noise because the information is irrelevant to the performance of the task. The classification task also involves a decision about whether there is a match or no match, and an orientation change can provide information for distinguishing the test pattern from the original. The difference must be ignored, however, in favor of attending to the relevant invariants which indicate a match. The greater the rotation of the test pattern relative to the memorized pattern, the more discriminable the two configurations, resulting in a monotonic increase in classification RT due to unnecessary attention to increasingly large rotation-dependent differences.

The increase in the latency of no-match responses relative to match responses between the practice session and the experimental session is also consistent with this explanation. In the practice session, during familiarization with the orientation changes used in the experiment, the effect of rotation-dependent differences was greater and led to premature no-match responses when the test form was a rotated positive set item. This explanation is partially supported by the slight increase in errors on match trials relative to errors on no-match trials, and the increase of these errors with degrees of rotation.

Regardless of the nature of the activity which deals with differences in orientation, the influence of rotational changes as a source of uncertainty or noise demands an interpretation different from Sternberg's (1967) noise condition, which reduced figure-ground contrast and resulted in an intercept increase. The increase in comparison time indicated that changes in orientation produced figure-figure uncertainty relative to matching relevant, rotation-independent invariants. As the $S$ learned with practice to attend only to the invariants, the slope effect dissipated and eventually disappeared, a change in comparison time which could serve as a strict criterion for shape constancy.

\section{REFERENCES}

Atkinson, R. C., \& Shiffrin, P. Human memory: A proposed system and its control processes. In $\mathrm{K}$. Spence and J. Spence (Eds.), The psychology of learning and motivation: Advances in research and theory. Vol. 2. New York: Academic Press. 1968.

Attneave, F., \& Arnoult, M. D. The quantitative study of shape and pattern perception. Psychological Bulletin, 1956, 53 . 452-471.

Checkosky, F. S. Speeded classification of multidimensional stimuli. Journal of Experimental Psychology, 1971, 87, 383-388.

Corballis, M. C., Kirby, J., \& Miller, A. Access to elements of a memorized list. Journal of Experimental Psychology, 1972, $94,185-190$

Hake. H. W. Contributions of psychology to the study of pattern vision. WADC Technical Report 57-621. [Republished in part as: Form discrimination and the invariance of form. In $L$. Uhr (Ed.), Pattern recognition. New York: Wiley, 1966.]

Posner, M. I., Boies, S. J., Eichelman, W. J., \& Taylor, R. C. Retention of visual and name codes of single letters. Journal of Experimental Psychology Monograph, 1969, 79, 1-16.

Shepard, R. N., \& Metzler, J. Mental rotation of three-dimensional objects. Science, 1971, 171, 701-703.

Sternberg, S. High speed scanning in human memory. Science, $1966,153,652-654$.

Sternberg, S. Two operations in character recognition. Perception \& Psychophysics, 1967, 2, 45-53.

Sternberg, S. Memory scanning: Mental processes revealed by reaction time experiments. American Scientist, 1969, 57, $421-457$.

Tversky, B. G. Pictorial and verbal encoding in a short-term memory task. Perception \& Psychophysics, 1969, 6, 225-233.

Wattenbarger, G. L. The representation of the stimulus in character classification. University of Michigan Technical Report No. 22, August 1970.

Woodring, A. V., \& Alluisi, E. A. Effects of choice figure rotation on the visual perception of form. Psychonomic Science, 1966, 4, 403-404.

\section{NOTE}

1. The deviant function for 0 -deg orientation in Session 1 appears to be an anomalous result. In a recent experiment, which utilized 0-, 45-, and 90-deg rotations, it was located appropriately below the 45 -deg function.

(Received for publication January 10, 1973; revision received March 22, 1973.) 